\title{
A NOTE ON LIPSCHITZ COMPACTIFICATIONS
}

\author{
JOUNI LUUKKAINEN
}

1. In [1] we introduced Lipschitz compactifications or LIP compactifications as compactifications in the category LIP of metric spaces and locally Lipschitz (LIP) maps. That is, if $f: X \rightarrow Y$ is a dense LIP embedding of a metric space $X$ into a compact metric space $Y$, then $Y$ or, more properly, the pair $(Y, f)$ is called a LIP compactification of $X$. Two LIP compactifications of $X$ are called LIP equivalent if they are equivalent through a lipeomorphism. We proved in $[1,1.14]$ that a metric space has a LIP compactification if and only if it is separable and locally totally bounded.

In this note we consider the problem whether a metrizable compactification $Z$ of a separable locally totally bounded metric space $X$ is equivalent to a LIP compactification of $X$, or equivalently, whether $Z$ can be metrized in such a way that $Z$ becomes a LIP compactification of $X$. Let $K_{Z}(X)$ denote the set of the LIP equivalence classes of the LIP compactifications of $X$ that are equivalent to $Z$. In Theorem 1 we give characterizations for $K_{Z}(X) \neq \emptyset$. Our main result, Theorem 2 , is that $K_{Z}(X) \neq \emptyset$ for every $Z$ if and only if $X$ is locally compact. In Theorem 3 we consider the cardinality of $K_{Z}(X)$. We now give an example where $K_{Z}(X)=\emptyset$. Let $X$ be the subspace $[0,1] \backslash\{1 / n \mid n \geqq 1\}$ of $\boldsymbol{R}$ and let $Y \subset \boldsymbol{R}$ be the union of $\{0\}$ and the intervals $(1 /(2 n+1), 1 / 2 n), n \geqq 1$. Then $\bar{X}$ and $Z=\bar{Y}$ are compact and there is a homeomorphism $f$ of $X$ onto $Y$ with $f(0)=0$. However, no neighborhood of 0 in $\bar{X}$ is homeomorphic to any neighborhood of 0 in $Z$. Hence the condition (2) of Theorem 1 is not satisfied and thus $K_{Z}(X)=\emptyset$.

For the undefined LIP terms we refer to [1].

2. A bijection $f$ between uniform spaces is called a locally uniform homeomorphism if both $f$ and $f^{-1}$ are locally uniformly continuous, i.e. uniformly continuous on some neighborhood of every point. We need the following modification of Lavrentiev's theorem [2, 24.9].

Lemma. Let $S$ and $T$ be complete Hausdorff uniform spaces, let $A \subset S$ and $B \subset T$ be dense subsets, and let $f: A \rightarrow B$ be a locally uniform homeomorphism. Then there are open sets $U \supset A$ and $V \supset B$ and a locally uniform homeomorphism $F: U \rightarrow V$ extending $f$. 
Proof. By [1, 2.9.5] $f$ and $f^{-1}$ have locally uniformly continuous extensions to open neighborhoods of $A$ and $B$, respectively. The proof can now be completed as in $[2,24.9]$.

Theorem 1. Let $X$ be a separable locally totally bounded metric space, let $f: X \rightarrow Z$ be a metrizable compactification of $X$, and let $\tilde{X}$ be the completion of $X$. Then the following conditions are equivalent:

(1) $K_{Z}(X) \neq \emptyset$.

(2) There are neighborhoods $U$ of $X$ in $\tilde{X}$ and $V$ of $f X$ in $Z$ and a homeomorphism $g: U \rightarrow V$ extending $f$.

(3) There are a neighborhood $U$ of $X$ in $\tilde{X}$ and an embedding $g: U \rightarrow Z$ extending $f$.

(4) There are a neighborhood $V$ of $f X$ in $Z$ and an embedding $h: V \rightarrow \tilde{X}$ extending $f^{-1}: f X \rightarrow X$.

(5) Consider $Z$ with its unique compatible uniformity, given by any compatible metric. Then $f$ defines a locally uniform homeomorphism of $X$ onto $f X$.

Proof. (1) $\Rightarrow(5)$ : Trivial.

$(5) \Rightarrow(3)$ and $(5) \Rightarrow(4)$ : This follows from the Lemma.

$(3) \Rightarrow(2)$ : By $[1,1.13] X$ has a locally compact neighborhood in $\tilde{X}$. Thus we may assume that $U$ is locally compact. Then $g U$ is locally compact and hence open in $Z$.

$(4) \Rightarrow(2)$ : This is proved as $(3) \Rightarrow(2)$.

$(2) \Rightarrow(1)$ : Let $e$ be the metric on $V$ for which $g: U \rightarrow(V, e)$ is an isometry. We may assume that $V$ is open. Then by $[1,6.4]$ there is a compatible metric $r$ on $Z$ which is LIP equivalent to $e$ on $V$. Hence $f: X \rightarrow(Z, r)$ is a LIP embedding.

Theorem 2. Let $X$ be a separable locally totally bounded metric space. Then $K_{Z}(X) \neq \emptyset$ for every metrizable compactification $Z$ of $X$ if and only if $X$ is locally compact.

Proof. Suppose that $X$ is locally compact and that $f: X \rightarrow Z$ is a metrizable compactification of $X$. Then $X$ is open in $\tilde{X}$ and $f X$ in $Z$. Hence the condition (2) of Theorem 1 is satisfied. Thus $K_{Z}(X) \neq \emptyset$.

Suppose now that $X$ is not locally compact. Then $X$ is not open in $\tilde{X}$. Hence by $[1,6.5]$ there is a compatible totally bounded metric $e$ on $X$ having no extension to a compatible metric on a neighborhood of $X$ in $\tilde{X}$. Then the completion $Z$ of $(X, e)$ is a compactification of $X$ such that the condition (2) of Theorem 1 is not satisfied. Thus $K_{Z}(X)=\emptyset$.

The sufficiency part of Theorem 2 generalizes the sufficiency part of a similar result $[1,1.6]$ on one-point compactifications and gives it a new proof.

In the next theorem we consider $K_{Z}(X)$ with its partial order which one gets through representatives setting $(Y, f) \leqq\left(Y^{\prime}, f^{\prime}\right)$ if there is a LIP map $g: Y^{\prime} \rightarrow Y$ with $g f^{\prime}=f$. 
Theorem 3. Let $X$ be a noncompact metric space and let $Z$ be a metrizable compactification of $X$ with $K_{\mathrm{Z}}(X) \neq \emptyset$. Then $K_{\mathrm{Z}}(X)$ has the cardinality of the continuum. In fact, $K_{\mathrm{Z}}(X)$ contains a subset which has the cardinality of the continuum and whose elements are not comparable.

Proof. We may assume that $Z$ is a compact metric space and that $X$ is a subspace of $Z$. Since $X \neq Z$, the proof can now be completed just as for one-point compactifications in the proof of $[1,1.9]$.

This generalizes [1, 1.9 and 1.10] and improves [1, 1.15.2].

3. Finally we consider the case where we allow the metric of $X$ to vary.

Theorem 4. Let $X$ be a metrizable space which is not locally compact, and let $f: X \rightarrow Z$ be a metrizable compactification of $X$. Then $X$ can be metrized by a totally bounded metric such that $K_{\mathrm{Z}}(X)=\emptyset$.

Proof. Since $f X$ is not open in $Z$, by $[1,6.5]$ there is a compatible totally bounded metric $e$ on $f X$ such that no compatible metric on $Z$ is LIP equivalent to $e$ on $f X$.

\section{References}

[1] Luukkainen, J.: Extension of spaces, maps, and metrics in Lipschitz topology. - Ann. Acad. Sci. Fenn. Ser. A I Math. Dissertationes 17, 1978, 1-62.

[2] Willard, S.: General topology. - Addison-Wesley Publishing Co., Reading, Mass. - Menlo Park, Calif.-London-Don Mills, Ont., 1970.

University of Helsinki

Department of Mathematics

SF-00100 Helsinki 10

Finland

Received 24 January 1980 